\title{
6. ROCKET AND SATELLITE INVESTIGATION OF METEORS
}

\author{
I. N. NAZAROVA
}

For recording meteor particles by means of rockets and satellites, we use equipment in the U.S.S.R. which permits not only the recording of the number of impacts but also the measuring of certain mechanical parameters of the meteor particles. Schematically speaking, the notion of a measuring device consists of the following:

From the viewpoint which interests us each particle may be characterized by its impulse and its energy (either mass or velocity). The impulse of a meteor particle when it strikes the barrier causes the measuring device to measure nothing, because the particle is exploded on the surface of the pick-up and the impulse on the pick-up of the material thrown out during the explosion considerably exceeds the impulse of the particle itself. Measuring this 'reactive' momentum perceptible to the pick-up, we obtain no impulse of the meteor particle, but apparently (with a certain scaling factor) its energy. A theoretical calculation, made by $K$. P. Stanyukovich, showed that for high velocities the momentum recorded by us was proportional to the energy of the striking particle. At present, theoretical investigations and laboratory experiments for determining this relation are being conducted. It is possible that the power exponent of the velocity in the expression $\frac{1}{2} m V^{2}$ for the energy may have to be decreased to obtain agreement.

Measurement of impulses would naturally be accomplished by a ballistic pick-up. Even though the collision time was negligible (of the order of $10^{-8} \mathrm{sec}$ ) it was expedient to select the natural frequency of the pick-up at around 400 cycles. The pick-up consists of a massive plate, suspended by a flat spring to which are connected four piezo-electric elements made of ammonium phosphate. Displacement of the plate, under the influence of the impact of a meteor particle, causes a deformation of the piezo-electric elements with the delivery of an electrical voltage in the form of short-period attenuating vibrations. These vibrations are separated according to amplitude by an amplifier-converter which computes the number of impulses in each amplitude range.

Our pick-ups measure impulses acting on their surfaces in the range $0 \cdot 1$ gcs to rooo gcs. Dispersion of readings in relation to the position of impact of the meteor particles does not exceed 10\%.

If one proceeds from the simplest theoretical law of the relation of the impulse received by the pick-up to the energy of the meteor particle and to the scaling factor of the impulse in energy (calculated by K. P. Stanyukovich, who assumed that the mean velocity of the meteor particle was equal to $40 \mathrm{~km} / \mathrm{sec}$ ), then the pick-ups employed can measure the energy of meteor particles with masses from $\mathrm{IO}^{-9} \mathrm{~g}$ and higher, as long as the pick-up holds out. The sensitivity of the system may be increased.

Thus, one of two values characterizing the mechanical properties of meteor particles can be successfully measured. It can be very important because erosion of the satellite surface also is connected with particle energies.

For recording meteor particles on the bottom of the satellite four pick-ups with a total area of $840 \mathrm{~cm}^{2}$ are installed. All pick-ups are situated in one plane. The altitude of the satellite in space, in so far as it is known from data of magnetic devices and solarorientation sensing elements, naturally determines the positions of our pick-ups in space.

During different times of flight a different frequency of impacts was recorded. This result cannot be attributed to a change in the number of meteor particles with changes in altitude, even though the satellite's altitude changed greatly, during its orbital flight, and even though simultaneously its position in space changed relative to the Earth, as the satellite rotated and precessed in various parts of the orbit, and even though the Earth at times partially screened the pick-ups from the striking of meteor particles.

For the three weeks during which the telemetering system operated on the satellite we obtained much material which is being processed at the present time.

According to preliminary data from the sensing elements and from the impacts on the $840 \mathrm{~cm}^{2}$ surface of the satellite, revolving about the Earth at an altitude of $1700-1880 \mathrm{~km}$, 


\section{JOINT DISCUSSION}

the average frequency of impacts was approximately equal to $7 \cdot 5 / \mathrm{sec}$, which corresponds to 90 impacts/ $\mathrm{I} \mathrm{m}^{2}$.

During partial shielding of pick-ups from the Earth at altitudes of $1300-1500 \mathrm{~km}$ and $500-600 \mathrm{~km}$, averages of 40 and $38 \mathrm{impacts} / \mathrm{sec} / \mathrm{m}^{2}$ respectively were recorded.

The spread at altitudes of $I 700-1880 \mathrm{~km}$ was $90 \pm 34$, at $1300-1500 \mathrm{~km}$ was $40 \pm 18$, and at $500-600 \mathrm{~km}$ was $38 \pm$ I0.

Proceeding from the assumption that the impulse detectable by the pick-up during the impact of a particle is proportional to its energy, then the meteor particles recorded have energies of the order of $10^{4}$ ergs.

The piezo-electric pick-ups for recording meteor particles are also mounted on the frame and head section of the rocket. Those mounted on rockets differ in design and sensitivity from those just described on the sputniks; the sensitivity of the rocket pickups was smaller.

During rocket ascents the impacts of meteor particles at altitudes from 150 to $300 \mathrm{~km}$ were reliably recorded. The frequency of impacts, on the average, was equal approximately to $2 \cdot 8 / \mathrm{sec}$ when the sensitive surface of the pick-ups was $900 \mathrm{~cm}^{2}$. Scaled to $\mathrm{I} \mathrm{m}^{2}$ area this corresponds to 31 impacts/sec.

\section{DIRECT MEASUREMENTS OF METEORITIC DUST USING ROCKETS AND SATELLITES}

\section{MAURICE DUBIN}

The space density and the mass distribution of meteoritic dust in the vicinity of the Earth may be directly measured using rocket and satellite vehicles. In comparison with optical and radio detection of meteors, rocket measurements are capable of detecting particles with diameters from $0 \cdot I$ to Io $\mu$. This range of particle sizes has been observed from photometric measurements of zodiacal light, by ground collections of deep-sea sediments and of the magnetic component of dust settling on flat surfaces, and collections from high-altitude aircraft. Direct measurements from rocket-launched vehicles appear the most reliable for identifying and detecting the meteoritic dust component: since meteor observations are not sensitive enough to detect this component, ground collections suffer from contamination of the terrestrial component of dust and variations resulting from hyper-velocity interactions with the atmosphere, while zodiacal light measurements are capable of determining the gross aspects of the cosmic dust in the ecliptic subject to assumptions of light scattering from solid particles.

Direct measurements of meteoritic dust from rocket-launched vehicles are also subject to a number of difficulties. In general, the limited time a rocket vehicle remains in the high atmosphere, even in the case of satellite vehicles, warrants the detection of the smallest component of meteoritic dust. This component has the greatest number density in space and hence allows one to obtain most easily a statistically valid sample of the dust component within the limited time that the detector is exposed. The impact of a micrometeorite upon an exposed surface results in the formation of a crater, a light flash, the ablation of surface material, and shock vibrations in the material. All of these effects have been utilized in various methods of detection of micro-meteorites from rocketlaunched vehicles. The main difficulties have been the hyper-velocity calibration to determine the absolute sensitivity of the detector, and the exposure of a sufficiently large surface long enough to obtain a good sampling free of contamination and interference.

A number of measurements of meteoritic dust have been made from rocket-launched vehicles using a hyper-sensitive accelerometer designed by Bohn. This method employs a piezo-electric crystal in contact with the surface of the rocket vehicle. This technique is effective for a wide range of sensitivities over a large exposed surface, yet is simple, compact, reliable, and permits telemetry of the detected impacts. The electrical pulse 